\title{
Methods of electron beam bunching
}

\author{
E.G.Bessonov ${ }^{\text {a }}$ \\ ${ }^{a}$ P.N. Lebedev Physical Institute RAS, 119991, Leninsky prospect 53, Moscow, Russia
}

A review of electron beam bunching methods is presented. A method to create trains of short electron microbunches by a creation of an electron multilayer mirror in the longitudinal phase space and then rotating it in fields of Radiofrequency (RF) accelerators or Free-Electron Laser (FEL) amplifiers is proposed.

\section{Introduction}

The production of short period trains of electron bunches (electron multilayer mirrors) is very important for the solution of the problem of compact, stable, monochromatic, diffraction limited prebunched FELs in the $\mathrm{mm}$ to X-ray regions.

Prebunched FELs were suggested by V.L.Ginsburg in 1947 and named "Sources of Coherent Undulator Radiation (UR)" [1]. The terms parametric and prebunched FELs appeared later [2]. H.Motz performed the first experiments on generation of coherent UR in 1953 3]. R.M.Phillips generated stimulated UR in a conventional FEL in the RF region in 1960 [4. In the early 1970's interest in prebunched FELs was renewed [5], [6].

Bunched electron beams of length $\sim 0.1 \div 1$ $\mathrm{mm}$ are produced in klystron bunchers (KB) and radio-frequency accelerators [7]. The basic components of a KB is an RF cavity followed by a drift space. The cavity is excited to the $T M_{010}$ mode. The electron beam at the exit of the buncher receives sinusoidal energy modulation. After the drift space the electron beam will be bunched due to the acquired velocity modulation.

A simple way to produce a bunched beam is to pass a continuous beam through a chopper, where the beam is deflected across a narrow slit or a system of slits, resulting in a pulsed beam behind the slit. The chopper includes an RF cavity excited like the buncher cavity but with the beam port offset by a distance from the cavity axis.

The chopper mode of bunching is rather wasteful. An RF buncher, which concentrates electrons from a large range of phases towards a particu- lar phase is more efficient for electron bunching. However, a chopper produces a beam with higher depth of modulation (clear bunches), does not introduce additional energy spread and can be used for specific reasons. Modern choppers work effectively in the RF region [8. Schemes of choppers for harder regions can be suggested (see below).

\section{Undulator bunchers}

Short-period trains of bunches are produced in undulator klystron bunchers (UKB). The energy modulation occurs in the undulator and electromagnetic wave fields of the UKB (FEL amplifier configuration) and bunching takes place in a free space [4]. The bunching length in UKB can be shortened if a dispersion section (chicane magnet) follows the undulator. Such a system was called an optical klystron buncher (OKB) $[9$.

The wavelength of the wave used in UKB is

$\lambda_{w}=\frac{\lambda_{u}}{2 \gamma_{s}^{2}}\left(1+p_{\perp}^{2}\right)$

where $p_{\perp}=\left.\sqrt{p_{\perp u}^{2}+p_{\perp w}^{2}}\right|_{p_{\perp w} \ll p_{\perp u}} \simeq p_{\perp u}$ $p_{\perp u(w)}=e B_{u(w)} \lambda_{u(w)} / 2 \pi m c^{2}$ are the transverse electron momenta determined by the electromagnetic fields of the undulator and the electromagnetic wave; $\lambda_{u}, B_{u}$ are the period and the magnetic field strength of the undulator; $B_{w}$, the magnetic field strength of the wave; $\gamma_{s}=$ $1 / \sqrt{1-\beta_{s}^{2}}=\sqrt{\lambda_{u}\left(1+p_{\perp}^{2}\right) / 4 \lambda_{w}}$, the relative electron equilibrium energy; $\beta_{s}=\sqrt{\beta_{\perp s}^{2}+\beta_{z s}^{2}}$; $\beta_{\perp s}=\beta_{\perp u s}+\beta_{\perp w s}=\left(p_{\perp u}+p_{\perp w}\right) / \gamma_{s} ; \beta_{z s}=$ $\lambda_{u} /\left(\lambda_{u}+\lambda_{w}\right) ; 2 \pi m c^{2} / e \simeq 10700 \mathrm{Gs} \cdot \mathrm{cm} ; \mathrm{e}, \mathrm{m}$, the electron charge and mass [10]. 
Undulators with high deflection parameters (wigglers) possess high dispersion. If the UKB works in the regime of high deflection parameter and high intensity of the wave (HHKB), the energy modulation and bunching can occur in the same undulator 10. In HHKB a high degree of bunching occurs if electrons produce a quarter of a period of phase oscillations. The bunching length in this case can be short $(\sim 1 \mathrm{~m}$ in the optical region, see section 3 ).

A scheme of bunching based on convergent waves and/or undulators with variable parameters (VPKB) was developed in 11. In this scheme an electron beam is trapped as a whole in regions less than those limited by separatrices of the FEL amplifier for about $3 / 4$ of a period of phase oscillations (see Fig. 1). Clear bunched beam can be used for high-efficiency generation on the fundamental or higher harmonics in the same undulator or in an undulator radiator. A similar scheme of bunching can occur in cases being developed in 12. 15.

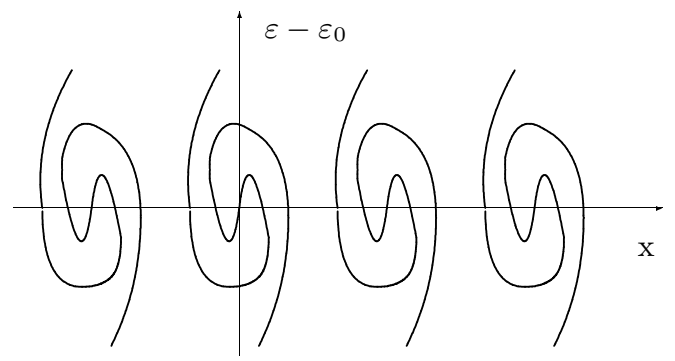

Fig. 1: Electron beam at the exit of VPKB in the phase space $\left(\varepsilon-\varepsilon_{0}, x\right)$. The amplitude of the laser electric field is $E_{w m}=E_{0}(1+\alpha z) . E_{0}=180 \mathrm{kV} / \mathrm{cm}$, $\alpha=0.8 \mathrm{~m}^{-1}, \lambda_{u}=1.5 \mathrm{~cm}$, the undulator length $L=5 \mathrm{~m}, B_{u}=3.10^{3} \mathrm{Gs}, \lambda_{w}=1.06 \mathrm{mkm}$.

A bunching scheme based on extraction of a series of microbunches from an electron beam located in storage ring buckets when an undulator with variable parameters and electromagnetic wave are used was considered in [16. Extracted microbunches stay in the storage ring buckets.

Shock acceleration of electron bunches or trains of bunches by wavepackets with a longitudinal component of electric field can lead to their compression. Such schemes can be realized in linear accelerators [17 and laser wake-field accelerators.

Below, a method of electron-beam bunching and schemes of realization are considered.

\section{A Method of Electron Beam Bunching}

The proposed method is based on production of electron beams in the form of trains of electron bunches, composed in the longitudinal plane in $N$ energy layers and followed by rotation of the bunches at a right angle 18. Rotation converts the bunches into short-period $\left(c T_{m b}<\lambda_{L} / N\right)$ trains of micro bunches with layers transverse to the beam propagation (see Fig. 2).

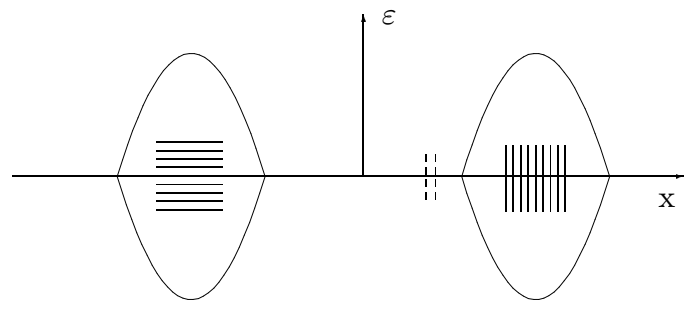

Fig. 2: An electron bunch arranged in energy layers in the left bucket is converted after its rotation in the right bucket to a train of micro bunches.

The longitudinal dimension of microbunches will be determined by the energy spread of layers and non linearity of phase oscillations. The influence of the non linearity can be neglected if the phase length of layers is short $(<\pi)$.

\subsection{Arrangement of electron beams with energy layers}

Example 1. A system of electron beams produced by $N$ electron guns of energy $\varepsilon_{n}=\varepsilon_{0}+$ $n \Delta \varepsilon_{l}$ can be arranged in one beam with energy layers by means of a bending magnet (see Fig. 3) [18. Here $n=0,1,2,3, \ldots N-1, \Delta \varepsilon_{l}$ is the interval between energy layers.

Example 2. A thin electron beam with finite energy spread and low transverse emittance passes through a dispersive magnet and a metal lattice with $N$ slits (see Fig. 4). After the lattice, the electron beam is arranged in $N$ energy layers ${ }^{1}$.

\footnotetext{
$\overline{{ }^{1} \text { More perfect systems }}$ of focusing bending magnets and
} 
If the electron beam has a small energy spread and an energy modulation, then this scheme can work in a chopper mode at RF, optical and harder regions.

If the ionization losses of electrons in the medium of a thin lattice are equal to $\Delta \varepsilon_{l} / 2$, then electrons of the incoming beam will be arranged in layers without losses.

If electron RF guns in Examples 1,2 produce short bunches, the beam at the exit of systems will be arranged in layers and bunched. The beam can be bunched at the exit of the bending magnet as well. After rotation at the bunching frequency, the beam will be a train of bunches a buncher's wavelength apart. Bunches will consist of $N$ microbunches (see Fig. 2).

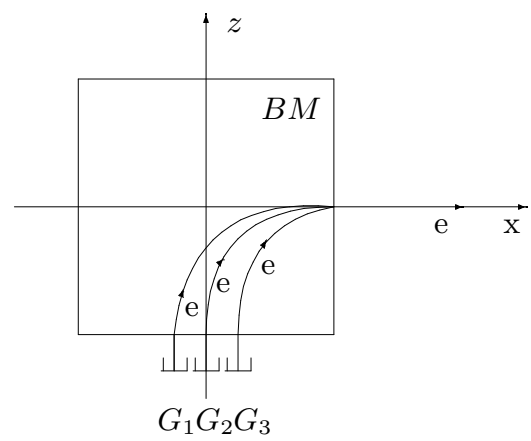

Fig. 3: The scheme of arranging an electron beam in energy layers. $G_{1}, G_{2}, G_{3}$ are the electron guns, $B M$ is the bending magnet.

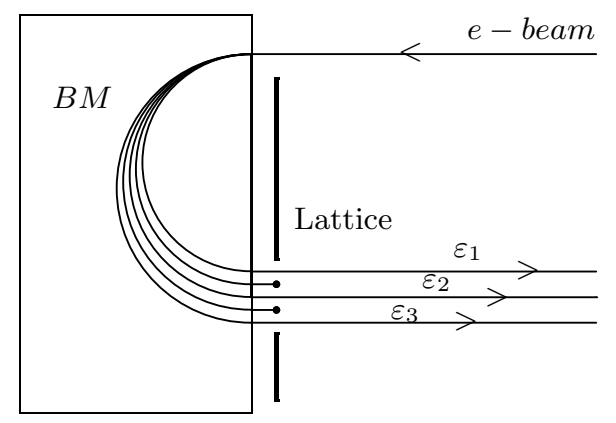

Fig. 4: The scheme of production of the electron beam arranged in layers. $B M$ is the bending magnet.

Example 3. Two counter-propagating short laser beams stored in an open resonator perform

quadrupole lenses can be used in this scheme. an interference lattice in a storage ring at a position of low-beta and high dispersion function. A low-emittance electron beam intersects the lattice at a 90 degree angle 19 .

In this case, the energy losses of electrons depend on their position in the laser beam. Electrons moving in the bright zones of fringes lose more energy than those in the dark zones. Their energy (closed orbits) will tend to the energy (closed orbits) of electrons in the nearest dark zones if the energy of scattered quanta $\hbar \omega_{\max } \ll$ $\Delta \varepsilon_{l}$. As a result, the electron beam will be arranged in energy layers if the interaction time will be much less than the period of phase oscillations of electrons in the storage ring.

More effective interaction of counterpropagating laser and electron beams can be produced in straight sections of storage rings if $T E M_{n, 0}$ laser mode is used $(n>1)$.

This scheme of bunching can be realized in ion storage rings using present-day technology (Rayleigh cross-section is $7 \div 10$ orders higher than Compton one) 18. Realization of the electron version of this scheme depends on the possibility of storing high-power laser beams in high-finesse optical resonators ${ }^{2}$.

\subsection{Rotation of electron beams in an FEL amplifier}

The frequency of phase oscillations of electrons in a helical undulator and a circular polarized laser wave $\omega_{\varphi}=\omega_{u} \sqrt{B_{u} B_{w}\left(1+\beta_{z s}\right)} / \beta_{z s} \gamma_{s} B_{c}$, where $\omega_{u}=2 \pi \beta_{z s} c / \lambda_{u} ; B_{c}=2 \pi m c^{2} / e \lambda_{u}[10]^{3}$.

Electrons produce a quarter of the period of phase oscillations inside the length

$$
\begin{aligned}
& l_{1 / 4}=\frac{\pi c \beta_{z s}}{2 \omega_{\varphi}}=\frac{\beta_{z s} \gamma_{s}}{4} \sqrt{\frac{\lambda_{u} \lambda_{w}}{\left(1+\beta_{z s}\right) p_{\perp u} p_{\perp w}}} \\
= & \frac{\lambda_{u}}{8 \sqrt{2}} \sqrt{\frac{1+p_{\perp}^{2}}{p_{\perp u} p_{\perp w}}} .
\end{aligned}
$$

${ }^{2}$ Note that prebunched Free-Ion Lasers present the ultimate in the capabilities of lasers 20. Relativistic ion beams can be cooled to a high degree in $6 \mathrm{D}$ space [21].

${ }^{3}$ We suppose that the laser beam intensity is constant along the interaction region of the laser and electron beams $\left(l_{1 / 4}<2 l_{R}\right.$, where $l_{R}=4 \pi \sigma_{L}^{2} / \lambda_{L}$ is the Rayleigh length). 
The maximum deviation of the electron energy in the bucket determined by a separatrix is

$$
\begin{array}{r}
\frac{\Delta \gamma_{s e p}}{\gamma_{s}}=\frac{2 \beta_{z s} \omega_{\varphi}}{\left(1+\beta_{z s}\right) \omega_{u}} \\
=4 \beta z s \sqrt{\frac{p_{\perp u} p_{\perp w}}{\left(1+\beta_{z s}\right)\left(1+p_{\perp}^{2}\right)}} .
\end{array}
$$

The magnetic field strength of the circular polarized Gaussian laser beam

$B_{w}[G s]=\sqrt{\frac{2 P_{w}}{c \sigma_{l}^{2}}} \simeq 2.58 \cdot 10^{-2} \sqrt{\frac{P[W]}{\sigma_{l}^{2}\left[c m^{2}\right]}}$.

Example 4. The parameters of the undulator and electromagnetic laser wave are the following:

$$
\begin{array}{lr}
\text { laser power } & P_{L}=1.6 \cdot 10^{9} \mathrm{~W}, \\
\text { laser wavelength } & \lambda=10^{-4} \mathrm{~cm}, \\
\text { laser beam dispersion } & \sigma_{L}=2 \cdot 10^{-2} \mathrm{~cm}, \\
\text { undulator magnetic field } & B_{u}=10700 \mathrm{Gs}, \\
\text { undulator period } & \lambda_{u}=3 \mathrm{~cm}, \\
\text { deflecting parameter } & p_{\perp}=3, \\
\text { Duration of laser wavepacket } \tau_{l}=2.5 \cdot 10^{-11} \mathrm{sec}, \\
\text { energy of laser wavepacket } \quad \varepsilon_{l}=P_{l} \tau_{l}=0.04 \mathrm{~J}, \\
\quad \begin{array}{l}
\text { In this case } \gamma_{s}=274, p_{\perp}=3, p_{\perp} w=4.82 \\
10^{-3}, l_{1 / 4}=69.7 \mathrm{~cm}, B_{w}=5.16 \cdot 10^{5} \mathrm{Gs}, l_{R}=1.0 \\
m,\left(2 l_{R}>l_{1 / 4}\right), \Delta \gamma_{s e p} / \gamma_{s}=1.07 \cdot 10^{-2} .
\end{array}
\end{array}
$$

\section{Conclusion}

Linear accelerator, storage ring and buncher technologies offer high-beam quality and short bunches necessary for operation of high efficiency, high-power, high-degree monochromatic prebunched FELs in optical and X-ray regions.

This work was supported partly by the Russian Foundation for Basic Research, Grant No 02-0216209.

\section{REFERENCES}

1. V.L.Ginzburg, Izv. Academy of Sciences USSR, Ser. Phys. 1947, V.11, No 2, p. 165 (in Russian).

2. E.G.Bessonov, Parametric Free - Electron Lasers, Nucl Instr. Meth., 1989, A282, p.442.

3. H.Motz, W.Torn, R.N.Whitehurst, J. Appl. Phys., 1953, V.24, No7, p.826.
4. R.M.Phillips, Trans. IRE.Electron Devices, 1960, V. 7, No. 4,p.231.

5. D.F.Alferov, Yu.A.Bashmakov, E.G.Bessonov, Sov. Phys. Tech. Phys. 1978, v.23, N8, part 1, p.902-904; part 2, p.905-909; Particle accelerators, 1979, v.9, No 4, p.223.

6. E.G.Bessonov, Proc. 4th General Conf. of the Europ. Phys. Soc., Chapter 7, 1979, York, UK, p.471.

7. B.E.Carlsten, D.W.Feldman, J.M.KinrossWright, Proc. of the MICRO BUNCHES WS, Upton, New York, Sept 1995, 21.

8. H.Wiedemann, Particle accelerator Physics, V.1, Springer-Verlag, 1998.

9. N.A.Vinokurov, A.N.Skrinsky, Preprint Inst. Nucl. Phys. No 77-59, Novosibirsk, 1977.

10. 6. D.F.Alferov, E.G.Bessonov, Preprint FIAN No 162, Moscow, 1977; Sov. Phys. Tech. Phys., 1979, V. 23, No 4, p. 450.

11. E.G.Bessonov, A.V.Serov, Preprint No 87, Lebedev Physical Institute, USSR, 1980; Sov. Phys. Tech. Phys. 1982, v.27, N2, 245.

12. J.Blau, T.Campbell, W.B.Colson, et al., Nucl. Instr. and Meth., A483 (2002), 142.

13. W.Colson, A.Todd, G.R.Neil, Proc. Int. Conf. on Free Electron Lasers 2001, M.Brunken, H.Genz, and A.Richter Editors, II-9.

14. A.V.Savilov, Nucl. Instr. and Meth., A483 (2002), 200.

15. G.N.Neil, L.Merminga, Rev. Modern Physics, V. 74, 2002, p. 685.

16. E.G.Bessonov, Proc. of 21st Internat. Free Electron Lasers Conf., Aug.23-28, 1999, Hamburg, Germany, II-51.

17. E.G.Bessonov, V.G.Kurakin, A.V.Serov, Sov. Phys. Tech. Phys., V.21, No 9, 1976, p. 1158; Doklady Academy Nauk USSR, v.280, No 4, 1985, p.843 (in Russian).

18. E.G.Bessonov, Proc. MICRO BUNCHES WS, Upton, New York, Sept 1995, 367.

19. T.Shintake, Nucl. Instr. and Meth., A311 (1992), 453.

20. E.G.Bessonov, Nucl. Instr. and Meth., A358 (1995), 204.

21. E.G.Bessonov, K.-Je Kim, Phys. Rev. Lett., V. 76, No 3, p. 431; Proc. 5th European Particle Accel. Conf., Sitges, Barcelona, 10-14 June 1995, v.2, p. 1196. 